\title{
Bounded-Distance Network Creation Games ${ }^{\star}$
}

\author{
Davide Bilò $^{1}$, Luciano Gualà ${ }^{2}$, and Guido Proietti ${ }^{3,4}$ \\ 1 Dip.to di Teorie e Ricerche dei Sistemi Culturali, University of Sassari, Italy \\ 2 Dipartimento di Matematica, University of Rome "Tor Vergata", Italy \\ 3 Dipartimento di Informatica, University of L'Aquila, Italy \\ 4 Istituto di Analisi dei Sistemi ed Informatica, CNR, Rome, Italy \\ E-mail: davide.bilo@uniss.it; guala@mat.uniroma2.it; guido.proietti@univaq.it
}

\begin{abstract}
A network creation game simulates a decentralized and non-cooperative building of a communication network. Informally, there are $n$ players sitting on the network nodes, which attempt to establish a reciprocal communication by activating, incurring a certain cost, any of their incident links. The goal of each player is to have all the other nodes as close as possible in the resulting network, while buying as few links as possible. According to this intuition, any model of the game must then appropriately address a balance between these two conflicting objectives. Motivated by the fact that a player might have a strong requirement about its centrality in the network, in this paper we introduce a new setting in which if a player maintains its (either maximum or average) distance to the other nodes within a given associated bound, then its cost is simply equal to the number of activated edges, otherwise its cost is unbounded. We study the problem of understanding the structure of associated pure Nash equilibria of the resulting games, that we call MAXBD and SumBD, respectively. For both games, we show that computing the best response of a player is an NP-hard problem. Next, we show that when distance bounds associated with players are non-uniform, then equilibria can be arbitrarily bad. On the other hand, for MAxBD, we show that when nodes have a uniform bound $R$ on the maximum distance, then the Price of Anarchy (PoA) is lower and upper bounded by 2 and $O\left(n \frac{1}{\frac{\left.\log _{3} R\right\rfloor+1}{1}}\right)$ for $R \geq 3$ (i.e., the PoA is constant as soon as the bound on the maximum distance is $\Omega\left(n^{\epsilon}\right)$, for some $\left.\epsilon>0\right)$, while for the interesting case $R=2$, we are able to prove that the $\mathrm{PoA}$ is $\Omega(\sqrt{n})$ and $O(\sqrt{n \log n})$. For the uniform SumBD we obtain similar (asymptotically) results, and moreover we show that the PoA becomes constant as soon as the bound on the average distance is $n^{\omega\left(\frac{1}{\sqrt{\log n}}\right)}$.
\end{abstract}

Keywords: Game Theory, NP-hardness, Nash Equilibria, Network Creation Game.

\footnotetext{
* This work was partially supported by the PRIN 2008 research project COGENT (COmputational and GamE-theoretic aspects of uncoordinated NeTworks), funded by the Italian Ministry of Education, University, and Research.
} 


\section{Introduction}

Communication networks are rapidly evolving towards a model in which the constituting components (e.g., routers and links) are activated and maintained by different owners, which one can imagine as players sitting on the network nodes. When these players act in a selfish way with the final intent of creating a connected network, the challenge is exactly to understand whether the pursuit of individual profit is compatible with the attainment of an equilibrium status for the system (i.e., a status in which players are not willing to move from), and how the social utility for the system as a whole is affected by the selfish behavior of the players. This task, which involves both computational and economical issues of the system, is exactly the aim of a research line which started with the seminal paper of Fabrikant et al. [9], where the by now classic network creation game (NCG) was initially formalized and investigated.

Definition of the NCG. In its original formulation, the NCG is defined as follows: We are given a set of $n$ players, say $V$, where the strategy space of player $v \in V$ is the power set $2^{V \backslash\{v\}}$. Given a combination of strategies $S=\left(S_{v}\right)_{v \in V}$, let $G(S)$ denote the underlying undirected graph whose node set is $V$, and whose edge set is $E(S)=\left\{\cup_{v \in V}\left(v \times S_{v}\right)\right\}$. Then, the cost incurred by player $v$ under $S$ is

$$
\operatorname{cost}_{v}(S)=\alpha \cdot\left|S_{v}\right|+\sum_{u \in V} d_{G(S)}(u, v)
$$

where $d_{G(S)}(u, v)$ is the distance between nodes $u$ and $v$ in $G(S)$. Thus, the cost function implements the inherently antagonistic goals of a player, which on the one hand attempts to buy as little edges as possible, and on the other hand aims to be as close as possible to the other nodes in the outcoming network. These two criteria are suitably balanced in (1) by making use of the parameter $\alpha \geq 0$. Consequently, the Nash Equilibria ${ }^{5}$ (NE) space of the game is heavily influenced by $\alpha$, and the corresponding characterization must be given as a function of it. The state-of-the-art for the Price of Anarchy (PoA) of the game, that we will call henceforth SuMNCG, is summarized in [13], where the most recent progresses on the problem have been reported.

Further NCG models. A criticism made to the classic NCG model is that the parameter $\alpha$ is in a sense exogenous to the system. Moreover, usage and building cost are summed up together in the player's cost, and this mixing is reflected in the social cost of the resulting network. As a consequence, we have that in this game the PoA alone does not say so much about the structural properties of the network, such as density, diameter, or routing cost. This gave rise to a sequence of new NCG models. A first natural variant of SUMNCG was introduced in [7], where the authors redefined the player cost function as follows

$$
\operatorname{cost}_{v}(S)=\alpha \cdot\left|S_{v}\right|+\max \left\{d_{G(S)}(u, v): u \in V\right\} .
$$

This variant, named MAXNCG, received further attention in [13], where the authors improved the PoA of the game on the whole range of values of $\alpha$. However, MAXNCG still incorporates in its definition the parameter $\alpha$. In an effort of defining new parameter-free models, in [12] the authors proposed an interesting variant in which a player $v$, when forming the network, has a limited budget $b_{v}$ to establish links to other players. This way, the player cost function restricts to the usage cost, namely either the maximum or the total

\footnotetext{
${ }^{5}$ In this paper, we only focus on pure strategies Nash equilibria.
} 
distance to other nodes. In particular, in [12] the authors focused on the latter measure. For this bounded-budget version of the game, that we call SUMBB, they showed that determining the existence of NE is NP-hard. On a positive side, they proved that for uniform budgets, say $k$, SumBB always admits a NE, and that its Price of Stability (PoS) is 1, while its PoA is $\Omega\left(\sqrt{\frac{n / k}{\log _{k} n}}\right)$ and $O\left(\sqrt{\frac{n}{\log _{k} n}}\right)$. Notice that in SumBB, links are seen as directed. Thus, a natural extension of the model was given in [8], were the undirected case was considered. For this, it was proven that both MAxBB and SumBB always admit a NE. Moreover, the authors showed that the PoA for MAxBB and SumBB is $\Omega(\sqrt{\log n})$ and $2^{O(\sqrt{\log n})}$, respectively, while in the special case in which the budget is equal to 1 for all the players, the PoA is $O(1)$ for both versions of the game.

In all the above models it must be noticed that, as stated in [9], for a player it is NPhard to find a best response once that the other players' strategies are fixed. To circumvent this problem, in [4] the authors proposed a further variant, called basic NCG (BNCG), in which given some existing network, the only improving transformations allowed are edge swaps, i.e., a player can only modify a single incident edge, by either replacing it with a new incident edge, or by removing it. This naturally induces a weaker concept of equilibrium for which a best response of a player can be computed in polynomial time. In this setting, the authors were able to give, among other results, an upper bound of $2^{O(\sqrt{\log n})}$ for the PoA of SumBNCG, and a lower bound of $\Omega(\sqrt{n})$ for the PoA of MAxBNCG. However, as pointed out in [13], the fact that now an edge has not a specific owner, prevents the possibility to establish any implications on the PoA of the classic NCG, since a NE in a BNCG is not necessarily a NE of a NCG.

Finally, another NCG model which is barely related to the NCG model we study in this paper has been addressed in [6].

Our results. In this paper, we propose a new NCG variant that complements the model proposed in [8]. More precisely, we assume that the cost function of each player only consists of the number of bought edges (without any budget on them), but with the additional constraint that a player $v$ needs to connect to the network by staying within a given (either maximum or average) distance, say (either $R_{v}$ or $D_{v}$ ), to the set of players. Our model is motivated by the fact that in a realistic scenario, a player might have a strong objective about its centrality in the created network, and this can only be guaranteed by means of our approach.

For this bounded-distance version of the NCG, we address the problem of understanding the structure of the NE associated with the two variants of the game, that we denote by MAXBD and SumBD. To this respect, we first show that both games can have an unbounded PoA as soon as players hold at least two different distance bounds. Moreover, in both games, computing a best response for a player is NP-hard. These bad news are counterbalanced by the positive result we get for uniform distance bounds. In this case, first of all, the PoS for MAXBD is equal to 1, while for SUMBD is at most equal to 2. Then, as far as the PoA is concerned, let $R$ and $D$ denote the uniform bound on the maximum and the average distance, respectively. We show that

(i) for MAxBD, the PoA is lower and upper bounded by 2 and $O\left(n^{\frac{1}{\left.\log _{3} R\right\rfloor+1}}\right)$ for $R \geq 3$, while for $R=2$ is $\Omega(\sqrt{n})$ and $O(\sqrt{n \log n})$; thus, the PoA is constant as soon as $R=\Omega\left(n^{\epsilon}\right)$, for some $\epsilon>0$;

(ii) for SumBD, the PoA is lower bounded by $2-\epsilon$, for any $\epsilon>0$, as soon as $D \geq 2-3 / n$, while it is upper bounded as reported in Table 1. 


\begin{tabular}{|l||c|c|c|c|c|}
\hline$D$ & $\in[2,3)$ & $\geq 3$ and $O(1)$ & $\omega(1) \cap O\left(3^{\sqrt{\log n}}\right)$ & $\omega\left(3^{\sqrt{\log n}}\right) \cap n^{O\left(\frac{1}{\sqrt{\log n}}\right)}$ & $n^{\omega\left(\frac{1}{\sqrt{\log n}}\right)}$ \\
\hline $\mathrm{PoA}$ & $O(\sqrt{n \log n})$ & $O\left(n^{\frac{1}{\left.\log _{3} D / 4\right]+2}}\right)$ & $2^{O(\sqrt{\log n})}$ & $O\left(n^{\frac{1}{\left.\log _{3} D / 4\right]+2}}\right)$ & $O(1)$ \\
\hline
\end{tabular}

Table 1. Obtained PoA upper bounds for SumBD.

The paper is organized as follows. After giving some basic definitions in Section 2, we provide some preliminary results in Section 3 . Then, we study upper and lower bounds for MAXBD and SumBD in Sections 4 and 5, respectively. Finally, in Section 6 we conclude the paper by discussing some intriguing relationships of our games with the famous graphtheoretic degree-diameter problem.

\section{Problem Definition}

Graph terminology. Let $G=(V, E)$ be an undirected (simple) graph with $n$ vertices. For a graph $G$, we will also denote by $V(G)$ and $E(G)$ its set of vertices and its set of edges, respectively. For every vertex $v \in V$, let $N_{G}(v):=\{u \mid u \in V \backslash\{v\},(u, v) \in E\}$. The minimum degree of $G$ is equal to $\min _{v \in V}\left|N_{G}(v)\right|$.

We denote by $d_{G}(u, v)$ the distance in $G$ from $u$ to $v$. The eccentricity of a vertex $v$ in $G$, denoted by $\varepsilon_{G}(v)$, is equal to $\max _{u \in V} d_{G}(u, v)$. The diameter and the radius of $G$ are equal to the maximum and the minimum eccentricity of its nodes, respectively. A node is said to be a center of $G$ if $\varepsilon_{G}(v)$ is equal to the radius of $G$. We define the broadcast cost of $v$ in $G$ as $B_{G}(v)=\sum_{u \in V} d_{G}(u, v)$, while the average distance from $v$ to a node in $G$ is denoted by $D_{G}(v)=B_{G}(v) / n$.

A dominating set of $G$ is a subset of nodes $U \subseteq V$ such that every node of $V \backslash U$ is adjacent to some node of $U$. We denote by $\gamma(G)$ the cardinality of a minimum cardinality dominating set of $G$. Moreover, for any real $k \geq 1$, the $k$ th power of $G$ is defined as the graph $G^{k}=\left(V, E\left(G^{k}\right)\right)$ where $E\left(G^{k}\right)$ contains an edge $(u, v)$ if and only if $d_{G}(u, v) \leq k$.

Let $U \subseteq V$ be a set of vertices, we denote by $G[U]$ the subgraph of $G$ induced by $U$. Let $F \subseteq\{(u, v) \mid u, v \in V, u \neq v\}$. We denote by $G+F$ (resp., $G-F$ ) the graph on $V$ with edge set $E \cup F$ (resp., $E \backslash F$ ). When $F=\{e\}$ we will denote $G+\{e\}$ (resp., $G-\{e\}$ ) by $G+e$ (resp., $G-e$ ). For two graphs $G_{1}$ and $G_{2}$, we denote by $G_{1} \cup G_{2}$ the graph with $V\left(G_{1} \cup G_{2}\right)=V\left(G_{1}\right) \cup V\left(G_{2}\right)$, and $E\left(G_{1} \cup G_{2}\right)=E\left(G_{1}\right) \cup E\left(G_{2}\right)$.

Problem statements. The bounded maximum distance NCG (MAXBD) is defined as follows: Let $V$ be a set of $n$ nodes, each representing a selfish player, and for any $v \in V$, let $R_{v}>0$ be an integer representing a bound on the eccentricity of $v$. The strategy of a player $v$ consists of a subset $S_{v} \subseteq V \backslash\{v\}$. Denoting by $S$ the strategy profile of all players, let $G(S)$ be the undirected graph with node set $V$, and with edge set $E(S)=\left\{\cup_{v \in V}\left(v \times S_{v}\right)\right\}$. When $u \in S_{v}$, we will say that $v$ is buying the edge $(u, v)$, or that the edge $(u, v)$ is bought by $v$. Then, the cost of a player $v$ in $S$ is $\operatorname{cost}_{v}(S)=\left|S_{v}\right|$ if $\varepsilon_{G(S)}(v) \leq R_{v},+\infty$ otherwise.

The bounded average distance NCG (SumBD) is defined analogously, with a bound $D_{v}$ on the average distance, and cost function $\operatorname{cost}_{v}(S)=\left|S_{v}\right|$ if $D_{G(S)}(v) \leq D_{v},+\infty$ otherwise. In the rest of the paper, depending on the context, we will interchangeably make use of the bound on the broadcast cost $B_{v}=D_{v} \cdot n$ when referring to SUMBD.

In both variants, we say that a node $v$ is within the bound in $S$ if $\operatorname{cost}_{v}(S)<+\infty$. We measure the overall quality of a graph $G(S)$ by its social cost $S C(S)=\sum_{v \in V} \operatorname{cost}_{v}(S)$. A graph $G(S)$ minimizing $S C(S)$ is called social optimum. 
We use the Nash Equilibrium (NE) as solution concept. More precisely, a NE is a strategy profile $S$ in which no player can decrease its cost by changing its strategy assuming that the strategies of the other players are fixed. When $S$ is a NE, we will say that $G(S)$ is stable, and that a graph $G$ is stable if there exists a strategy profile $S$ such that $G=G(S)$. Notice that in both games, when $S$ is a NE, all nodes are within the bound and, since every edge is bought by a single player, $S C(S)$ coincides with the number of edges of $G(S)$.

We conclude this section by recalling the definition of the two measures we will use to characterize the NE space of our games, namely the Price of Anarchy (PoA) [9] and the Price of Stability $(\mathrm{PoS})$ [3], which are defined as the ratio between the highest (respectively, the lowest) social cost of a NE and the cost of a social optimum.

\section{Preliminary results}

First of all, observe that for MAXBD it is easy to see that a stable graph always exists. Indeed, if there is at least one node having distance bound 1, then the graph where all 1-bound nodes buy edges towards all the other nodes is stable. Otherwise, any spanning star is stable. Notice that any spanning star is stable for SumBD as well, but only when all vertices have a bound of at least $2 n-3$, while the problem of understanding whether a NE always exists for the remaining values is open. From these observations, we can derive the following negative result:

Theorem 1. The PoA of MAxBD and SumBD (with distance bounds $B_{v} \geq 2 n-3$ ) is $\Omega(n)$, even for only two distance-bound values.

Proof. We will define a graph $G$ with $\Omega\left(n^{2}\right)$ edges, and we will prove that $G$ is stable for both versions of the game. Then, we will show that in both cases the cost of the social optimum is $n-1$.

The graph $G$ is defined as follows. We have a clique of $k$ nodes. For each node $v$ of the clique, we add four nodes $v_{1}^{1}, v_{2}^{1}, v_{1}^{2}, v_{2}^{2}$ and four edges $\left(v_{2}^{1}, v_{1}^{1}\right),\left(v_{1}^{1}, v\right),\left(v_{2}^{2}, v_{1}^{2}\right),\left(v_{1}^{2}, v\right)$. Clearly, $G$ has $n=5 k$ nodes and $\Omega\left(n^{2}\right)$ edges. Now, consider a strategy profile $S$ with $G=G(S)$ and such that (i) every edge is bought by a single player, and (ii) the edges $\left(v_{2}^{j}, v_{1}^{j}\right),\left(v_{1}^{j}, v\right)$ are bought by $v_{2}^{j}$ and $v_{1}^{j}$, respectively, $j=1,2$. Now, we show that $S$ is a $\mathrm{NE}$, once we have defined suitable bounds for the players.

Let us consider MAXBD first. We set the bound of every node of the clique to 3, while all the other nodes have bound 5. Trivially, all nodes are within the bound. Moreover, a node $v_{i}^{j}$ is buying only one edge and, since the removal of such edge disconnects the graph, $v_{i}^{j}$ cannot decreases its cost. Let $v$ be a node of the clique, and assume that $v$ is buying $h$ edges in $S$. Let $S^{\prime}$ be a strategy profile where $v$ switches its strategy $S_{v}$ with $S_{v}^{\prime}$ and such that $\left|S_{v}^{\prime}\right|<h$. Since $h \leq n-1$, it must exist a vertex $u$ of the clique such that $u \in S_{v}$ and $u, u_{1}^{1}, u_{2}^{1}, u_{1}^{2}, u_{2}^{2} \notin S_{v}^{\prime}$, from which we have that $v$ cannot be within the bound in $S^{\prime}$ since $d_{G\left(S^{\prime}\right)}\left(v, u_{2}^{2}\right)>3$.

Concerning SumBD, we set the bound of each node $v$ of the clique to $\sum_{u \in V} d_{G}(v, u)=$ $11 k-5>2 n-3$, while we assign to all the other nodes bound $n^{2}$. Similar arguments used for MAXBD can be used to show that $S$ is a NE for SUMBD as well.

To conclude the proof, observe that any star (with cost $n-1$ ) is a social optimum for the two instances of MAXBD and SumBD given above.

Given the above bad news, from now on we focus our attention on the uniform case of the games, i.e., that in which all the bounds on the distances are the same, say $R$ and $D$ (i.e., $B=D \cdot n$ ) for the maximum and the average version, respectively. Similarly to other 
NCGs, also here we have the problem of computing a best response for a player, as stated in the following theorem.

Theorem 2. Computing the best response of a player in MAXBD and SUMBD is NPhard.

Proof. Let us consider MAXBD first. The reduction is from the NP-hard minimum dominating set problem which, given a graph $G^{\prime}=\left(V^{\prime}, E^{\prime}\right)$, asks for finding a dominating set of $G^{\prime}$ of minimum cardinality, say $\gamma\left(G^{\prime}\right)$. Let $N=\left|V^{\prime}\right|$. We build a graph $G$ with $n=N+2 N(R-2)+1$ vertices as follows: We have an isolated vertex $u$, a copy of $G^{\prime}$, and two paths of length $R-2$ appended to every vertex $v \in V^{\prime}$. Now, let $S$ be the strategy profile such that $G=G(S)$. Clearly, $\operatorname{cost}_{u}(S)=+\infty$, and it is easy to see that $u$ has a strategy yielding a cost of $k$ if and only if $\gamma\left(G^{\prime}\right) \leq k$.

Now, for SumBD, we sketch a reduction from the $k$-median problem. Let $G^{\prime}=\left(V^{\prime}, E^{\prime}\right)$ be an instance of the $k$-median problem which, given a value $\beta$, asks for finding a subset $U \subseteq V$ of size $k$ such that $\sum_{v \in V} \min _{u \in U} d_{G}(u, v) \leq \beta$. This problem is NP-hard even when $G^{\prime}$ is an unweighted graph [10]. Let $G$ be the graph defined as $G^{\prime}$ with an additional isolated node $u$, and let $S$ be a strategy profile such that $G=G(S)$, and let $B=\beta+N$, where $N=\left|V^{\prime}\right|$. It is easy to see that $u$ has a strategy yielding a cost of $k$ if and only if $G^{\prime}$ has a $k$-median of cost at most $\beta$.

On the other hand, a positive result which clearly implies that SUMBD always admits a pure NE is the following.

Theorem 3. The PoS of MAXBD is 1, while for SumBD is at most 2.

Proof. Concerning MAXBD, when $R=1$ the complete graph is a social optimum as well as the only stable graph. For $R>1$, let $T$ be a spanning star with center $c \in V$ and edges $(c, v), v \in V \backslash\{c\}$. Clearly, $T$ is a social optimum, and the strategy profile $S$ in which $S_{v}=\{c\}$ for every $v \in V \backslash\{c\}$, and $S_{c}=\emptyset$, is a NE.

Concerning SumBD, observe that such a $T$ is an optimum as well as stable when $B \geq 2 n-3$. Now assume that $B=n-1+k$ with $0 \leq k \leq n-2$. We will define a graph $G$ with a number of edges that is at most twice the number of edges of the optimum, and we show that it is stable. Let $h, t \geq 0$ be s.t. $n=(k+1) h+t$. We partition $V$ into $h$ groups of $k+1$ nodes, say $V_{1}, \ldots, V_{h}$ and, when $t \neq 0$, an additional group $V_{0}$ of $t$ vertices. The edge set of $G$ is defined as $\left\{(u, v) \mid u \in V_{i}, v \in V_{j}, i \neq j\right\}$. Let $S$ be a strategy profile such that $G=G(S)$ with the constraint that every node in $V_{0}$ buys no edge in $S$. Clearly, every node in $G$ is within the bound. Moreover, observe that in order to be within the bound, each node $v$ must have degree at least $n-1-k$. Now, since every node not in $V_{0}$ has degree exactly $n-1-k$, and since nodes in $V_{0}$ buy no edges, then $G(S)$ is stable. To bound the social cost of $G$, notice that the cost of the optimum, say Opt, is at least $\frac{n(n-1-k)}{2}$. Let us consider the case in which $k<n / 2$. Then, Орт $\geq n^{2} / 4$, while $S C(S) \leq n^{2} / 2 \leq 2$. Орт. On the other hand, when $k \geq n / 2$, we have only two groups, one with $t=n-k-1$ nodes and the other with $n-t$. Then, we have $S C(S)=t(n-t) \leq t n \leq 2 \cdot$ Opt.

We conclude this section by providing a lemma which will simplify the exposition of the remaining results.

Lemma 1. Let $G(S)$ be a stable graph and let $H$ be a subgraph of $G(S)$. If for each node $v$ there exists a set $E_{v}$ of edges (all incident to $v$ ) such that $v$ is within the bound in $H+E_{v}$, then $S C(S) \leq|E(H)|+\sum_{v \in V}\left|E_{v}\right|$. 
Proof. Let $k_{v}$ be the number of edges of $H$ that $v$ is buying in $S$. If $v$ buys $E_{v}$ additionally to its $k_{v}$ edges, then $v$ will be within the bound. Hence, since $S$ is a NE, we have that $\operatorname{cost}_{v}(S) \leq k_{v}+\left|E_{v}\right|$, from which it follows that:

$$
S C(S)=\sum_{v \in V} \operatorname{cost}_{v}(S) \leq \sum_{v \in V} k_{v}+\sum_{v \in V}\left|E_{v}\right|=|E(H)|+\sum_{v \in V}\left|E_{v}\right| .
$$

\section{PoA for MaxBD}

\subsection{Upper bounds}

Lemma 2. Let $G(S)$ be a $N E$, and let $\gamma$ be the cardinality of a minimum dominating set of $G(S)^{R-1}$, then $S C(S) \leq(\gamma+1)(n-1)$.

Proof. Let $U$ be a minimum dominating set of $G(S)^{R-1}$, with $\gamma=|U|$. It is easy to see that there is a spanning forest $F$ of $G(S)$ consisting of $\gamma$ trees $T_{1}, \ldots, T_{\gamma}$, such that every $T_{j}$ contains exactly one vertex in $U$, and when we root $T_{j}$ at such vertex the height of $T_{j}$ is at most $R-1$.

For a node $v \in V$, let $E_{v}=\{(v, u) \mid u \in U \backslash\{v\}\}$. Clearly, $v$ is within the bound in $F+E_{v}$, hence by using Lemma 1 , we have

$S C(S) \leq|E(F)|+\sum_{u \in U}\left|E_{u}\right|+\sum_{v \in V \backslash U}\left|E_{v}\right|=n-\gamma+(\gamma-1) \gamma+\gamma(n-\gamma) \leq(\gamma+1)(n-1)$.

Let $G(S)$ be a NE and let $v$ be a node of $G(S)$. Since $v$ is within the bound, the neigborhood of $v$ in $G$ is a dominating set of $G^{R-1}$. Therefore, thanks to Lemma 2 we have proved the following corollary.

Corollary 1. Let $G(S)$ be a $N E$, and let $\delta$ be the minimum degree of $G(S)$, then $S C(S) \leq$ $(\delta+1)(n-1)$.

We are now ready to prove our upper bound to the PoA for the game.

Theorem 4. The PoA of MAXBD is $O\left(n^{\frac{1}{\left\lfloor\log _{3} R\right\rfloor+1}}\right)$ for $R \geq 3$, and $O(\sqrt{n \log n})$ for $R=2$.

Proof. Let $G$ be a stable graph, and let $\gamma$ be the size of a minimum dominating set of $G^{R-1}$. We define the ball of radius $k$ centered at a node $u$ as $\beta_{k}(u)=\left\{v \mid d_{G}(u, v) \leq k\right\}$. Moreover, let $\beta_{k}=\min _{u \in V}\left|\beta_{k}(u)\right|$. The idea is to show that in $G$ the size of any ball increases quite fast as the radius of the ball increases.

Claim. For any $k \geq 1$, we have $\beta_{3 k+1} \geq \min \left\{n, \gamma \beta_{k}\right\}$.

Proof. Consider the ball $\beta_{3 k+1}(u)$ centered at any given node $u$, and assume that $\left|\beta_{3 k+1}(u)\right| \leq$ $n$. Let $T$ be the maximal set of nodes at distance exactly $2 k+1$ from $u$ and subject to the distance between any pair of nodes in $T$ being at least $2 k+1$. We claim that for every node $v \notin \beta_{3 k+1}(u)$, there is a vertex $t \in T$ with $d_{G}(t, v)<d_{G}(u, v)$. Indeed, consider the node $t^{\prime}$ in the shortest path between $v$ and $u$ at distance exactly $2 k+1$ from $u$. If $t^{\prime} \in T$ the claim 
trivially holds, otherwise consider the node $t \in T$ that is closest to $t^{\prime}$. From the maximality of $T$ we have that $d_{G}(t, v) \leq d_{G}\left(t, t^{\prime}\right)+d_{G}\left(t^{\prime}, v\right) \leq 2 k+d_{G}(u, v)-(2 k+1)<d_{G}(u, v)$.

As a consequence, we have that $T \cup\{u\}$ is a dominating set of $G^{R-1}$, and hence $|T|+1 \geq \gamma$. Moreover, all the balls centered at nodes in $T \cup\{u\}$ with radius $k$ are all pairwise disjoint. Then:

$$
\left|\beta_{3 k+1}(u)\right| \geq\left|\beta_{k}(u)\right|+\sum_{t \in T}\left|\beta_{k}(t)\right| \geq \gamma \beta_{k} .
$$

Now, observe that since the neighborhood of any node is a dominating set of $G^{R-1}$, we have that $\beta_{1} \geq \gamma$. Then, after using the above claim $x$ times, we obtain

$$
\beta_{\frac{3^{x+1}-1}{2}} \geq \min \left\{n, \gamma^{x+1}\right\} .
$$

Let us consider the case $R \geq 3$ first. Let $U$ be a maximal independent set of $G^{R-1}$. Since $U$ is also a dominating set of $G^{R-1}$, it holds that $|U| \geq \gamma$. We consider the $|U|$ balls centered at nodes in $U$ with radius given by the value of the parameter $x=\left\lfloor\log _{3} R-1\right\rfloor$. Every ball has radius at most $(R-1) / 2$ and since $U$ is an independent set of $G^{R-1}$, all balls are pairwise disjoint and hence we have $n \geq|U| \gamma^{\left\lfloor\log _{3} R-1\right\rfloor+1} \geq \gamma^{\left\lfloor\log _{3} R\right\rfloor+1}$. As a consequence, we obtain $\gamma \leq n^{\frac{1}{\left.\log _{3} R\right\rfloor+1}}$, and the claim now follows from Lemma 2 .

Now assume $R=2$. We use the bound given in [5] to the size $\gamma(G)$ of a minimum dominating set of a graph $G$ with $n$ nodes and minimum degree $\delta$, namely $\gamma(G) \leq \frac{n}{\delta+1} H_{\delta+1}$, where $H_{i}=\sum_{j=1}^{i} 1 / j$ is the $i$-th harmonic number. Hence, since a social optimum has cost $n-1$, from Lemma 2 and Corollary 1 , we have $\frac{S C(S)}{n-1} \leq \min \left\{\delta+1, \frac{n}{\delta+1} H_{\delta+1}+1\right\}$, for any stable graph $G(S)$ with minimum degree $\delta$. The claim follows.

\subsection{Lower bounds}

We first prove a simple constant lower bound for any value of $R=o(n)$, and then we show an almost tight lower bound of $\Omega(\sqrt{n})$ for $R=2$. We postpone to the concluding section a discussion on the difficulty of finding better lower bounds for large values of $R$.

Theorem 5. For any $\epsilon>0$ and for $1<R=o(n)$, the PoA for MAXBD is at least $2-\epsilon$.

Proof. Assume we are given a set of $n=2 R+h$ vertices $\left\{u_{1}, \ldots, u_{2 R}\right\} \cup\left\{v_{1}, \ldots, v_{h}\right\}$. The strategy profile $S$ is defined as follows. Vertex $u_{j}$ buys a single edge towards $u_{j+1}$, for each $j=1, \ldots, 2 R-1$, and every $v_{i}$ buys two edges towards $u_{1}$ and $u_{2 R}$. It is easy to see that $G(S)$ has diameter $R$ and is stable. The claim follows from the fact that $S C(S)$ goes to $2(n-1)$ as $h$ goes to infinity and the fact that, as observed in Section 3, a spanning star (having social cost equal to $n-1$ ) is a social optimum.

We close this section by providing a much stronger lower bound for the special case in which $R=2$. Before stating the theorem, we give some additional notation. Let $v$ be a player, $S$ be a strategy profile, and $\ell$ a positive integer. We define $N_{S}^{\ell}(v)=\{u \mid u \in$ $\left.V, d_{G(S)}(u, v) \leq \ell\right\}$ and $\bar{N}_{S}^{\ell}(v)=V \backslash N_{S}^{\ell}(v)$. We will omit the superscript $\ell$ when $\ell=1$. Moreover, we denote by $\gamma(S, v, \ell)$ the size of a minimum cardinality set $X \subseteq V$ of vertices that dominates $\bar{N}_{S}^{\ell}(v)$ in $G(S)^{\ell-1}$, i.e., for every vertex $u \in \bar{N}_{S}^{\ell}(v)$ there exists a vertex $x \in X$ such that $d_{G(S)}(x, u) \leq \ell-1$, i.e., $(x, u) \in E\left(G(S)^{\ell-1}\right)$. Finally, denote by $S_{\neg v}$ the 
strategy profile where each player but $v$ plays the same strategy as in $S$, while $v$ buys no edge, i.e., the strategy of $v$ is $\emptyset$. The following proposition, whose proof is straightforward, provides exact bounds to the cost incurred by each player in every connected graph, and will be used in the proof of the theorem.

Proposition 1. Let $G(S)$ be a connected graph. The cost incurred by each player $v$ in $S$ in MAXBD with bound $R$ is $\left|S_{v}\right| \geq \gamma\left(S_{\neg v}, v, R\right)$. Moreover, a player $v$ is in equilibrium in $S$ iff $\left|S_{v}\right|=\gamma\left(S_{\neg v}, v, R\right)$.

Let $S^{\prime}$ be a strategy profile for a set of players $V$. A strategy profile $S$ for $V$ extends $S^{\prime}$ if $S_{v}^{\prime} \subseteq S_{v}$ for every $v \in V$. Let $S$ and $S^{\prime}$ be two strategy profiles for a set of players $V$ such that $S$ extends $S^{\prime}$, For every $v \in V$ let $N\left(S^{\prime}, S, v\right)=N_{S}(v) \backslash N_{S^{\prime}}(v)$ and let $S^{v, S^{\prime}}$ be the strategy profile such that $S_{v}^{v, S^{\prime}}=S_{v}^{\prime} \cup N\left(S^{\prime}, S, v\right)$ and $S_{u}^{v, S^{\prime}}=S_{u}^{\prime} \cup\left\{v^{\prime} \mid v^{\prime} \in S_{u}, v^{\prime} \neq v\right\}$ for each $u \in V, u \neq v$. Observe that $G\left(S^{v, S^{\prime}}\right)=G(S)$.

The following proposition will be also used in the proof of the theorem.

Proposition 2. Let $V$ be a set of players and let $S, S^{\prime}$ be two strategy profiles for $V$ such that $S$ extends $S^{\prime}$. If every player $v$ is in equilibium in $S^{v, S^{\prime}}$, then $G(S)$ is a stable graph.

Proof. For the sake of contradiction, assume that every player $v \in V$ is in equilibium in $S^{v, S^{\prime}}$ but $G(S)$ is not stable. Then there exists a player $u$ and a strategy profile $S^{\prime \prime}$ such that (i) $S_{v}^{\prime \prime}=S_{v}$ for every $v \in V, v \neq u$, (ii) the eccentricity of $u$ in $G\left(S^{\prime \prime}\right)$ is less than or equal to $R$, and (iii) $\left|S_{u}^{\prime \prime}\right|<\left|S_{u}\right|$. Let $X=\left\{x \mid x \in S_{u}^{\prime \prime}, x \notin S_{u}\right\}$ and let $Y=\left\{y \mid y \in S_{u}, y \notin S_{u}^{\prime \prime}\right\}$. By (iii) we have $|X|<|Y|$. Let $\bar{S}$ be the strategy profile such that $\bar{S}_{u}=\left(S_{u}^{u, S^{\prime}} \backslash Y\right) \cup X$ and $\bar{S}_{v}=S_{v}^{u, S^{\prime}}$ for every $v \in V, v \neq u$. Clearly, $G\left(S^{\prime \prime}\right)=G(\bar{S})$ and thus, by (ii) the eccentricity of $u$ in $G(\bar{S})$ is less than or equal to 2 . Furthermore, $|X|<|Y|$ implies $\left|\bar{S}_{u}\right| \leq\left|S_{u}^{u, S^{\prime}}\right|$ and therefore $u$ is not in equilibrium in $S^{u, S^{\prime}}$.

We are now ready to prove the following.

Theorem 6. The PoA of MAxBD for $R=2$ is $\Omega(\sqrt{n})$.

Proof. Let $p \geq 3$ be a prime number. We provide a graph $G^{\prime}$ of diameter 2 containing $O\left(p^{2}\right)$ vertices and $\Omega\left(p^{3}\right)$ edges and show that there exists a strategy profile $S$ such that $G(S)=G^{\prime}$ and $G(S)$ is stable. $G^{\prime}$ contains two vertex-disjoint rooted trees $T$ and $T^{\prime}$ as subgraphs. $T$ is a complete $p$-ary tree of height 2 . We denote by $r$ the root of $T$, by $C=\left\{c_{0}, \ldots, c_{p-1}\right\}$ the set of children of $r$, and by $V_{i}=\left\{v_{i, 0}, \ldots, v_{i, p-1}\right\}$ the set of children of $c_{i} . T^{\prime}$ is a star with $p^{2}$ leaves rooted at the center $r^{\prime}$. The leaves of $T^{\prime}$ are partitioned in $p$ groups each having exactly $p$ vertices. For every $i=0, \ldots, p-1$, we denote by $U_{i}=\left\{u_{i, 0}, \ldots, u_{i, p-1}\right\}$ the set of vertices of group $i . G^{\prime}=(V, E)$ has vertex set $V=V(T) \cup V\left(T^{\prime}\right)$ and edge set (see also Figure 1)

$$
\begin{aligned}
E & =E(T) \cup E\left(T^{\prime}\right) \cup\left\{\left(r, r^{\prime}\right)\right\} \\
& \cup\left\{\left(c, c^{\prime}\right) \mid c, c^{\prime} \in C, c \neq c^{\prime}\right\} \\
& \cup \bigcup_{i=0}^{p-1}\left\{\left(u, u^{\prime}\right) \mid u, u^{\prime} \in U_{i}, u \neq u^{\prime}\right\} \\
& \cup\left\{\left(u_{i, j}, v_{i^{\prime}, j^{\prime}}\right) \mid i, i^{\prime}, j, j^{\prime} \in[p-1], j+i^{\prime} i \equiv j^{\prime} \quad(\bmod p)\right\} .
\end{aligned}
$$

We claim that the diameter of $G^{\prime}$ is 2 . The eccentricity of $r$ is 2 as $T$ has height $2, T^{\prime}$ has height 1 , and $G^{\prime}$ contains the edge $\left(r, r^{\prime}\right)$. Observe that the subgraphs of $G^{\prime}$ induced 


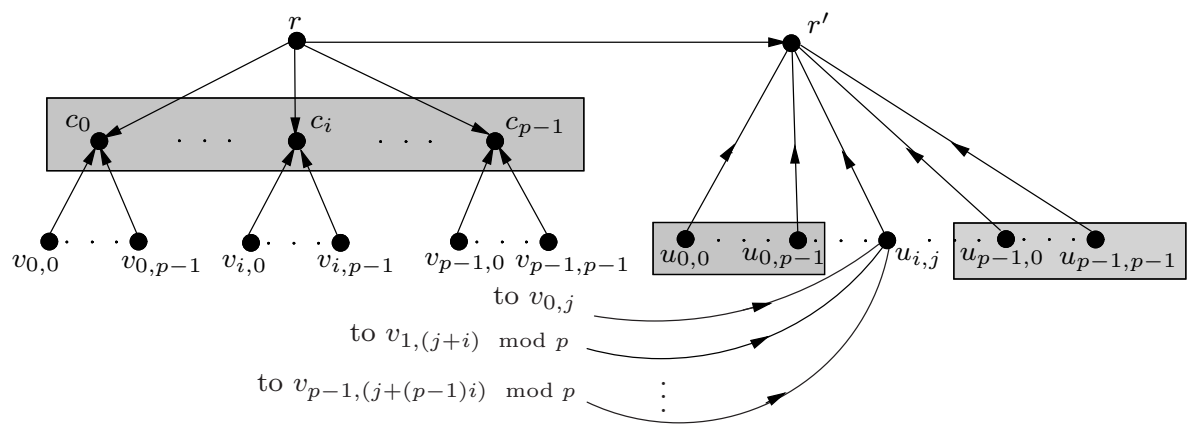

Fig. 1. The graph $G(S)$. Edges are bought from the nodes they exit from. Notice that nodes in grey boxes are clique-connected (with arbitrary orientations), and for the sake of readability we have only inserted edges leading to node $u_{i, j}$.

by $C$ and $U_{i}$, for all $i \in[p-1]$, are all cliques of $p$ vertices. Furthermore, by construction, there is an edge linking each vertex $u \in \bar{U}$ with some $v \in V_{i}$, for every $i \in[p-1]$, and thus $V_{i}$ dominates $\bar{U}$. Therefore, the eccentricity of each vertex in $C$ is 2 . As a consequence, to prove that $G^{\prime}$ has diameter 2 , it is enough to prove that

(i) $U_{i}$ dominates $\bar{V}$, for every $i \in[p-1]$ (so as each vertex in $\bar{U}$ would have eccentricity 2),

(ii) for every pair $v \in V_{i}$ and $v^{\prime} \in V_{i^{\prime}}, i, i^{\prime} \in[p-1], i \neq i^{\prime}$, there is a vertex $u \in \bar{U}$ such that $(v, u),\left(v^{\prime}, u\right) \in E$ (so as each vertex in $\bar{V}$ would have eccentricity 2 ).

To prove (i), simply observe that for every $i^{\prime}, j^{\prime} \in[p-1]$, there always exists a $j \in[p-1]$ such that $j+i^{\prime} i \equiv j^{\prime}(\bmod p)$, and thus, $\left(u_{i, j}, v_{i^{\prime}, j^{\prime}}\right) \in E$. To prove (ii), observe that for every $v_{i, j}, v_{i^{\prime}, j^{\prime}} \in \bar{V}$, with $i \neq i^{\prime}$, there always exists $i^{\prime \prime}, j^{\prime \prime} \in[p-1]$ such that $j^{\prime \prime}+i i^{\prime \prime} \equiv j$ $(\bmod p)$ and $j^{\prime \prime}+i^{\prime} i^{\prime \prime} \equiv j^{\prime}(\bmod p)$ as $p$ is a prime number (simply choose $i^{\prime \prime}$ such that $\left.i^{\prime \prime}\left(i-i^{\prime}\right) \equiv\left(j-j^{\prime}\right)(\bmod p)\right)$. Therefore, $\left(v_{i, j}, u_{i^{\prime \prime}, j^{\prime \prime}}\right),\left(v_{i^{\prime}, j^{\prime}}, u_{i^{\prime \prime}, j^{\prime \prime}}\right) \in E$.

To complete the proof, it remains to show that there exists a strategy profile $S$ such that $G(S)=G^{\prime}$ and $G(S)$ is stable. Let $\bar{V}$ and $\bar{U}$ be the set of leaves of $T$ and $T^{\prime}$, respectively. Let $S^{\prime}$ be a strategy profile where:

- each vertex in $\bar{V} \cup\{r\}$ buys all edges incident to it (thus, each vertex in $\bar{V} \cup\{r\}$ buys exactly $p+1$ edges),

- each vertex in $\bar{U}$ buys the edge towards $r^{\prime}$,

- each of the remaining vertices buys no edge, i.e., $S_{v}^{\prime}=\emptyset$ for every $v \in C \cup\left\{r^{\prime}\right\}$.

Let $S$ be any strategy profile that extends $S^{\prime}$ such that $G(S)=G^{\prime}$. Observe that

- $r^{\prime}$ buys no edge in $S^{r, S^{\prime}}$,

- each vertex $v$ in $C \cup \bar{U}$ buys exactly $p$ edges in $S^{v, S^{\prime}}$,

- each vertex $v$ in $\bar{V} \cup\{r\}$ buys exactly $p+1$ edges in $S^{v, S^{\prime}}$.

First of all observe that

$$
\left|\bar{N}_{S_{\neg v v}^{v, S^{\prime}}}^{2}(v) \cap \bar{V}\right|= \begin{cases}0 & \text { if } v=r^{\prime} \\ p^{2} & \text { if } v=r ; \\ p^{2}-1 & \text { if } v \in \bar{V} \\ p(p-1) & \text { otherwise. }\end{cases}
$$


Let $\hat{V} \subseteq \bar{V}$ be such that $|\hat{V}| \in\left\{p^{2}, p^{2}-1, p(p-1)\right\}$, and let $X$ be a set of vertices that dominates $\hat{V}$ in $G(S)$.

Claim. $|X| \geq\left\lceil\frac{|\hat{V}|}{p}\right\rceil$ where equality holds only if $X \subseteq C$ or $X \subseteq \bar{U}$.

Proof. Let $X$ be a set of vertices that dominates $\hat{V}$ in $G(S)$ and observe that $X \subseteq \hat{V} \cup C \cup \bar{U}$. As any vertex of $G(S)$ can dominate at most $p$ vertices of $\hat{V}$, we have that $|X| \geq\left\lceil\frac{|\hat{V}|}{p}\right\rceil$.

Now we prove that if $|X|=\left\lceil\frac{|\hat{V}|}{p}\right\rceil$ then either $X \subseteq C$ or $X \subseteq \bar{U}$. Indeed, any set $X^{\prime}$ dominating $\hat{V}$ in $G(S)$ and containing any vertex of $\hat{V}$ has size $\left|X^{\prime}\right| \geq 1+\left\lceil\frac{|\hat{V}|-1}{p}\right\rceil>\left\lceil\frac{|\hat{V}|}{p}\right\rceil$, where the first inequality holds because any vertex in $\hat{V}$ dominates only itself while the second inequality holds by the choice of $|\hat{V}|$ and because $p \geq 3$. Furthermore, any set $X^{\prime}$ containing $0<k<\left\lceil\frac{|\hat{V}|}{p}\right\rceil$ vertices of $C$ and $\left\lceil\frac{|\hat{V}|}{p}\right\rceil-k$ vertices of $\bar{U}$ can dominate at most $p k+p\left(\left\lceil\frac{|\hat{V}|}{p}\right\rceil-k\right)-k\left(\left\lceil\frac{|\hat{V}|}{p}\right\rceil-k\right)=p\left\lceil\frac{|\hat{V}|}{p}\right\rceil-k\left\lceil\frac{|\hat{V}|}{p}\right\rceil+k^{2} \leq p\left\lceil\frac{|\hat{V}|}{p}\right\rceil-\left\lceil\frac{|\hat{V}|}{p}\right\rceil+1 \leq|\hat{V}|-1$ vertices of $\hat{V}$ (and so $X^{\prime}$ cannot dominate $\hat{V}$ ), where the last inequality holds by the choice of $|\hat{V}|$ and because $p \geq 3$.

As a consequence of the above claim, if $|X|=\left\lceil\frac{|\hat{V}|}{p}\right\rceil$, i.e., either $X \subseteq C$ or $X \subseteq \bar{U}$, then either $X$ does not dominate $r$ in $G(S)$ or $X$ does not dominate $r^{\prime}$ in $G(S)$. This implies that,

$$
\gamma\left(S_{\neg v}^{v, S^{\prime}}, v, 2\right)= \begin{cases}0 & \text { if } v=r^{\prime} \\ p & \text { if } v \in C \cup \bar{U} \\ p+1 & \text { otherwise. }\end{cases}
$$

Since $\left|S_{v}^{v, S^{\prime}}\right|=\gamma\left(S_{\neg v}^{v, S^{\prime}}, v, 2\right)$ for each player $v \in V$, from Proposition 1 we have that $v$ is in equilibrium w.r.t. $S^{v, S^{\prime}}$. Therefore, by Proposition $2, G(S)$ is stable.

\section{SumBD}

\subsection{Upper bounds}

For SumBD, we start by giving an upper bound to the PoA similar to the one obtained for MAXBD. For the remaining of this section we use $D$ to denote the average bound of every node, namely $D=B / n$.

Theorem 7. The PoA of SumBD is $O\left(n^{\frac{1}{\left.\log _{3} D / 4\right]+2}}\right)$ for $D \geq 3$, and $O(\sqrt{n \log n})$, when $2 \leq D<3$.

Proof. Let $G=G(S)$ be a stable graph, and let $\rho=S C(S) /(n-1)$. Remind that the ball of radius $k$ centered at a node $u$ is defined as $\beta_{k}(u)=\left\{v \mid d_{G}(u, v) \leq k\right\}$. Moreover, let $\beta_{k}=\min _{u \in V}\left|\beta_{k}(u)\right|$. We have the following

Claim. For any $k \geq 1$, we have $\beta_{3 k+2} \geq \min \left\{n / 2+1,\lfloor\rho\rfloor \beta_{k}\right\}$.

Proof. Consider the ball $\beta_{3 k+2}(u)$ centered at any given node $u$, and assume that $\left|\beta_{3 k+2}(u)\right| \leq$ $n / 2$. Let $T$ be the maximal set of nodes at distance exactly $2 k+2$ from $u$ and subject to the distance between any pair of nodes in $T$ being at least $2 k+1$. We claim that for every node $v \notin \beta_{3 k+2}(u)$, there is a vertex $t \in T$ with $d_{G}(t, v) \leq d_{G}(u, v)-2$. Indeed, consider the node 
$t^{\prime}$ in the shortest path between $v$ and $u$ at distance exactly $2 k+2$ from $u$. If $t^{\prime} \in T$ the claim trivially holds, otherwise consider the node $t \in T$ that is closest to $t^{\prime}$. From the maximality of $T$ we have that $d_{G}(t, v) \leq d_{G}\left(t, t^{\prime}\right)+d_{G}\left(t^{\prime}, v\right) \leq 2 k+d_{G}(u, v)-(2 k+2) \leq d_{G}(u, v)-2$.

Let $H$ be the forest consisting of the following disjoint trees. For every node $t \in T \cup\{u\}$, let $U_{t}$ be the nodes that are closer to $t$ than any other $t^{\prime} \in T \cup\{u\}$, and let $F_{t}$ be the subtree of the shortest path tree of $G$ rooted at $t$ spanning $U_{t}$. As a consequence, since $u$ is within the bound in $G$, it is easy too see that every vertex $x$ is within the bound in $H \cup\{(x, t) \mid t \in(T \cup\{u\}) \backslash\{x\}\}$. Hence, From Lemma 1, we have that $\rho<|T|+1$ and hence $|T|+1 \geq\lfloor\rho\rfloor$. Now, all the balls centered at nodes in $T \cup\{u\}$ with radius $k$ are all pairwise disjoint. Then:

$$
\left|\beta_{3 k+2}(u)\right| \geq\left|\beta_{k}(u)\right|+\sum_{t \in T}\left|\beta_{k}(t)\right| \geq\lfloor\rho\rfloor \beta_{k} .
$$

Now, observe that $\beta_{1} \geq\lfloor\rho\rfloor$. Then, after using the above claim $x$ times, we obtain

$$
\beta_{23^{x}-1} \geq \min \left\{n / 2+1,\lfloor\rho\rfloor^{x+1}\right\} .
$$

Let us consider the case $R \geq 3$ first. Let $U$ be a maximal independent set of $G^{D-1}$. Since $U$ is also a dominating set of $G^{D-1}$, it holds that $|U| \geq\lfloor\rho\rfloor$. We consider the $|U|$ balls centered at nodes in $U$ with maximal radius at most $(D-2) / 2$. Since $U$ is an independent set of $G^{D-1}$, all balls are pairwise disjoint and hence we have $n \geq|U|\lfloor\rho\rfloor^{\left\lfloor\log _{3} D / 4\right\rfloor+1} \geq$ $\lfloor\rho\rfloor^{\left.\log _{3} D / 4\right\rfloor+2}$. As a consequence, we obtain $\lfloor\rho\rfloor \leq n^{\frac{1}{\left.\log _{3} D / 4\right\rfloor}}$, and the claim follows.

Now assume $2 \leq D<3$. To use the same argument used for MAxBD, it suffices to prove that for any stable graph $G(S)$ with minimum degree $\delta$, it holds that $\frac{S C(S)}{n-1} \leq \min \{\delta+$ $\left.1, O\left(\gamma\left(G^{D-1}\right)\right)\right\}$. The upper bound $\frac{S C(S)}{n-1}=O\left(\gamma\left(G^{D-1}\right)\right)$ can be proved by using the same arguments used in the proof of Lemma 2 where we exchange the role of $R$ with $D$. Now, we prove that $\frac{S C(S)}{n-1} \leq \delta+1$. Let $v$ be a node with degree $\delta$, and let $N_{G(S)}(v)=\left\{u_{1}, \ldots, u_{\delta}\right\}$. Consider a shortest path tree $T$ of $G(S)$ rooted at $v$. Clearly, $v$ is within the bound in $T$, and if we define $E_{x}=\left\{\left(x, u_{j}\right) \mid 1 \leq j \leq \delta\right\}$ for any $x \neq v$, we have $B_{T+E_{x}}(x) \leq B_{G(S)}(v) \leq B$. Hence, from Lemma 1, if follows that $S C(S) \leq|E(T)|+(n-1) \delta \leq(\delta+1)(n-1)$.

From the above result, it follows that the PoA becomes constant when $D=\Omega\left(n^{\epsilon}\right)$, for some $\epsilon>0$. We now show how to lower such a threshold to $D=2^{\omega(\sqrt{\log n})}=n^{\omega\left(\frac{1}{\sqrt{\log n}}\right)}$ (and we also improve the upper bound when $D=\omega(1) \cap o\left(3^{\sqrt{\log n}}\right)$ ).

Lemma 3. Let $G(S)$ be stable and let $v$ be a node such that $B_{G(S)}(v) \leq B-n$, then $S C(S) \leq 2(n-1)$.

Proof. Let $T$ be the shortest path of $G$ rooted at $v$. The claim immediately follows from Lemma 1 by observing that $v$ is within the bound in $T$ and every other node $u$ is within the bound in $T+(u, v)$.

Notice that the above Lemma shows that when a stable graph $G$ has diameter at most $D-1$ then the social cost of $G$ is at most twice the optimum. Now, the idea is to provide an upper bound to the diameter of any stable graph $G$ as function of $\delta$, where $\delta$ is minimum degree of $G$. Then we combine this bound with Lemma 3 in order to get a better upper bound to PoA for interesting ranges of $D$.

The proof of the following theorem follows the schema of that of Theorem 9 in [4]. 
Theorem 8. Let $G$ be stable with minimum degree $\delta$. Then the diameter of $G$ is $2^{O(\sqrt{\log n})}$ if $\delta=2^{O(\sqrt{\log n})}$, and $O(1)$ otherwise.

Proof. We start by proving two lemmas:

Lemma 4. Let $G$ be stable with minimum degree $\delta$. Then either $G$ has diameter at most $2 \log n$ or, for every node $u$, there is a node $x$ with $d_{G}(u, x) \leq \log n$ such that (i) $x$ is buying $\delta / c$ edges (for some constant $c>1$ ), and (ii) the removal of these edges increases the sum of distances from $x$ by at most $2 n(1+\log n)$.

Proof. Assume that the diameter of $G$ is greater than $2 \log n$ and consider a node $u$. Let $U_{j}$ be the set of nodes at distance exactly $j$ from $u$ and let $n_{j}=\left|U_{j}\right|$. Moreover, denote by $T$ the shortest path tree of $G$ rooted at node $u$. Let $i$ be the minimum index such that $n_{i+1}<2 n_{i}(i$ must exist since the height of $T$ is greater than $\log n)$. Consider the set of edges $F$ of $G$ having both endpoints in $U_{i-1} \cup U_{i} \cup U_{i+1}$ and that do not belong to $T$. Then, $|F| \geq \delta n_{i} / 2-3 n_{i}$. Moreover, we have that $n_{i-1}+n_{i}+n_{i+1} \leq 1 / 2 n_{i}+n_{i}+2 n_{i}=7 / 2 n_{i}$. As a consequence, there is a vertex $x \in U_{i-1} \cup U_{i} \cup U_{i+1}$ which is buying at least $\frac{n_{i} / 2-3 n_{i}}{7 / 2 n_{i}} \geq \delta / c$ edges of $F$, for some constant $c>1$. Moreover, when $x$ removes these edges, the distance to any other node $y$ increases by at most $2(1+\log n)$ because $d_{T}(x, y) \leq 2(1+\log n)$. The claim follows.

Lemma 5. In any stable graph $G$, there is a constant $c^{\prime}>1$ the addition of $\delta / c^{\prime}$ edges all incident to a node $u$ decreases the sum of distances from $u$ by at most $5 n \log n$.

Proof. If $G$ has diameter at most $2 \log n$, then the claim trivially holds. Otherwise, let $x$ be the node of the previous Lemma and let $c^{\prime}$ be such that $\delta / c^{\prime} \leq \delta / c-1$. Moreover, assume by contradiction that the sum of distances from $u$ decreases by more than $5 n \log n$ when we add to $G$ the following set of edges $F=\left\{\left(u, v_{1}\right), \ldots,\left(u, v_{h}\right)\right\}$, with $h=\delta / c^{\prime}$. Then, let $F^{\prime}=\left\{\left(x, v_{j}\right) \mid j=1, \ldots, h\right\}$. We argue that $x$ can improves his cost by saving at least an edge as follows: $x$ deletes its $\delta / c$ edges and adds $F^{\prime}$. Indeed, the sum of distances from $x$ increases by at most $2 n(1+\log n) \leq 4 n \log n$ and decreases by at least $5 n \log n-n \log n$, since for every node $y$ such that the shortest path in $G+F$ from $u$ to $y$ passes through $x$, we have that $d_{G}(u, y)-d_{G+F}(u, y) \leq \log n$. Hence, $x$ is still within the bound in $G+F^{\prime}$ and is saving at least one edge: a contradiction.

Recall that the ball of radius $k$ centered at a node $u$ is defined as $\beta_{k}(u)=\left\{v \mid d_{G}(u, v) \leq\right.$ $k\}$. Moreover, let $\beta_{k}=\min _{u \in V}\left|\beta_{k}(u)\right|$. We claim that

$$
\beta_{4 k} \geq \min \left\{n / 2+1, \frac{k \delta}{20 c \log n} \beta_{k}\right\},
$$

for some constant $c>1$. To prove that, consider the ball $\beta_{4 k}(u)$ centered at any given node $u$, and assume that $\left|\beta_{4 k}(u)\right| \leq n / 2$. Let $T$ be the maximal set of nodes at distance exactly $2 k+1$ from $u$ and subject to the distance between any pair of nodes in $T$ being at least $2 k+1$. It is easy to see that, from the maximality of $T$, for every node $v \notin \beta_{3 k}$ there is a node $t \in T$ such that $d_{G}(v, t) \leq d_{G}(u, v)-k$. We assumed that at least $n / 2$ nodes have distance more than $3 k$. This implies that there must be a set $T^{\prime} \subseteq T$ of size $\delta / c$ such that at least $n \delta / 2|T|$ such vertices $v$ whose distance is at most $d(u, v)-k$ from some node in $T^{\prime}$. If we add $\delta / c$ edges from $u$ to nodes in $T^{\prime}$, the sum of distances from $u$ decreases by at least $(k-1) n / 2|T| \geq k n / 4|T|$. By Lemma 5 this improvement is at most $5 n \log n$. As a consequence we have that $|T| \geq \delta k /(20 c \log n)$. Moreover, all the balls centered at nodes in $T$ are disjoint, and this proves (3). Now, the claim follows by solving the recurrence (3). 
By using the above theorem along with Lemma 3, and observing that if $G(S)$ is stable and has minimum degree $\delta$, then $\frac{S C(S)}{n-1} \leq \delta+1$, as shown in the proof of Theorem 7 , we have:

Theorem 9. The PoA of SumBD is $2^{O(\sqrt{\log n})}$ if $D=\omega(1)$, and $O(1)$ if $D=2^{\omega(\sqrt{\log n})}$.

Then, by combining the results of Theorems 7 and 9, we get the bounds reported in Table 1.

\subsection{Lower bounds}

We can finally prove the following theorem.

Theorem 10. For any $\epsilon>0$ and for $2 n-3 \leq B=o\left(n^{2}\right)$, the PoA of SUMBD is at least $2-\epsilon$.

Proof. To prove the theorem, we use the following scheme. First, for every integer $k \geq 2$, we provide a family $\mathcal{G}_{k}$ of graphs that are stable when $B \in\left[\lambda(k, n), \lambda^{\prime}(k, n)\right)$, where $n$ is the size of the graph and $\lambda(k, n)$ and $\lambda^{\prime}(k, n)$ are functions that depend on $k$ and $n$. We also prove that the social cost of infinitely many graphs in $\mathcal{G}_{k}$ is at least $2-\epsilon$ far from the social cost of an optimum, for every $k=o(n)$. Then, we show that $\lambda(2, n) \leq 2 n-3$, $\lambda(k+1, n) \leq \lambda^{\prime}(k, n)$, and $\lambda(\Omega(n), n)=\Omega\left(n^{2}\right)$.

Family $\mathcal{G}_{k}$ contains a graph $G_{k, h}$ for every positive integer $h$. More precisely, $G_{k, h}$ has $n_{k, h}=(h+1) k$ vertices and $m_{k, h}=2 k h$ edges. Therefore, the lower bound of $2-\epsilon$ for the PoA when $k=o(n)$ follows by choosing $h \geq \frac{2}{\epsilon}-1$. For the rest of the proof, we assume that $h$ is an arbitrary, but fixed, positive integer. Moreover, with a little abuse of notation, we will drop the subscript $h$ from $G_{k, h}$ and $n_{k, h}$ and we will also drop the parameter $n_{k}=n_{k, h}$ as argument of the two functions $\lambda$ and $\lambda^{\prime}$.

The graph $G_{k}$ is a highly symmetric graph consisting of $k$ players $\left\{u_{0}, \ldots, u_{k-1}\right\}$ which buy no edge, and, for every $i=0, \ldots, k-1$, there are $h$ copies of a player (we denote by $v_{i}$ any of such players) each buying exactly two edges: one towards $u_{i}$ and one towards $u_{i+1 \bmod k}$. Observe that $G_{k}$ has diameter $k$.

The broadcast cost of each player $v_{i}$ is exactly $\lambda(k)$ while the broadcast cost of each player $u_{j}$ is equal to $\bar{\lambda}(k)$. It is easy to see that $\lambda(2)=2 n_{2}-4, \bar{\lambda}(2)=n_{2}$.

Moreover, one can observe that for every $k \geq 2$

$$
\lambda(k+1)=\lambda(k)+n_{k}+ \begin{cases}h & \text { if } k+1 \text { is even } \\ 1 & \text { if } k+1 \text { is odd }\end{cases}
$$

as well as

$$
\bar{\lambda}(k+1)=\bar{\lambda}(k)+n_{k}+ \begin{cases}1 & \text { if } k+1 \text { is even } \\ h & \text { if } k+1 \text { is odd }\end{cases}
$$

As each player $v_{i}$ owns exactly two edges, the only strategy $v_{i}$ has to connect to $G_{k}-v_{i}$ with exactly one edge, is that of connecting either to some $v_{i}^{\prime}$ or to some $u_{j}$. Therefore, a lower bound on the broadcast cost of player $v_{i}$ if he uses only one edge to connect to $G_{k}-v_{i}$ is $\lambda^{\prime}(k)=\min \{\lambda(k), \bar{\lambda}(k)\}+n_{k}-1-k$, as $G_{k}$ has diameter $k$. Therefore, we have that $G_{k}$ is stable for every $B \in\left[\max \{\lambda(k), \bar{\lambda}(k)\}, \lambda^{\prime}(k)\right)$. In what 
follows, we show that $\max \{\lambda(k), \bar{\lambda}(k)\}=\lambda(k)$, thus proving that $B \in\left[\lambda(k), \lambda^{\prime}(k)\right)$, as well as $\lambda^{\prime}(k)=\bar{\lambda}(k)+n_{k}-1-k$.

Indeed, for every $k \geq 2$, and using $n_{k+1}=n_{k}+h+1$, we have that

$$
\lambda(k+2)=\lambda(k)+2 n_{k+1} \quad \text { and } \quad \bar{\lambda}(k+2)=\bar{\lambda}(k)+2 n_{k+1} .
$$

Furthermore, using the relations $n_{k+1}=n_{k}+h+1, n_{k}=(h+1) k$, and the formulas above, $\lambda(3)=2 n_{3}-3, \bar{\lambda}(3)=\frac{5}{3} n_{3}-1$. Therefore, $\bar{\lambda}(2) \leq \lambda(2)$ and $\bar{\lambda}(3) \leq \lambda(3)$. As a consequence, for every $k \geq 2, \bar{\lambda}(k+2)=\bar{\lambda}(k)+2 n_{k+1} \leq \lambda(k)+2 n_{k+1}=\lambda(k+2)$. Therefore $\max \{\lambda(k), \bar{\lambda}(k)\}=\lambda(k)$.

To complete the proof, it remains to show that $\lambda(2) \leq 2 n_{2}-3, \lambda(k+1) \leq \lambda^{\prime}(k)$, and $\lambda(\Omega(n))=\Omega\left(n^{2}\right)$. We already proved that $\lambda(2)=2 n_{2}-4 \leq 2 n_{2}-3$. Moreover, for $n_{k}=2 k$, i.e., $k=n_{k} / 2$, we have that $G_{k}$ is a cycle of $2 k$ vertices, and thus the broadcast cost of any vertex is $\Omega\left(k^{2}\right)=\Omega\left(n^{2}\right)$. Finally, using induction, and observing that $\lambda(3) \leq \lambda^{\prime}(2)$, we can prove that $\lambda(k+1) \leq \lambda^{\prime}(k)$. Indeed, if $k+1$ is even, then

$$
\begin{aligned}
\lambda(k+1) & =\lambda(k)+n_{k}+h \leq \lambda^{\prime}(k-1)+n_{k}+h \\
& =\bar{\lambda}(k-1)+n_{k-1}+n_{k}-1-k+h \\
& =\bar{\lambda}(k)+n_{k}-1-k=\lambda^{\prime}(k),
\end{aligned}
$$

while, if $k+1$ is odd, then

$$
\begin{aligned}
\lambda(k+1) & =\lambda(k)+n_{k}+1 \leq \lambda^{\prime}(k-1)+n_{k}+1 \\
& =\bar{\lambda}(k-1)+n_{k-1}+n_{k}-1-k+1 \\
& =\bar{\lambda}(k)+n_{k}-1-k=\lambda^{\prime}(k) .
\end{aligned}
$$

\section{Concluding remarks}

In this paper, we have introduced a new NCG model in which the emphasis is put on the fact that a player might have a strong requirement about its centrality in the resulting network, as it may well happen in decentralized computing (where, for instance, the bound on the maximum distance could be used for synchronizing a distributed algorithm). We developed a systematic study on the PoA of the two (uniform) games MAxBD and SumBD, which, however, needs to be continued, since a significant gap between the corresponding lower and upper bounds is still open. In particular, it is worth to notice that finding a better upper bound to the PoA would provide a better estimation about how much dense a network in equilibrium can be.

Actually, in an effort of reducing such a gap, we focused on MAXBD, and we observed the following fact: Recall that a graph is said to be self-centered if every node is a center of the graph (thus, the eccentricity of every node is equal to the radius of the graph, which then coincides with the diameter of the graph). An interesting consequence of Lemma 2 is that only stable graphs that are self-centered can be dense, as one can infer from the following

Proposition 3. Let $G(S)$ be a $N E$ for MAxBD such that $G(S)$ is not self-centered. Then, $S C(S) \leq 2(n-1)$. 
Proof. Let $v$ be a node with minimum eccentricity. It must be that $\varepsilon_{G(S)}(v) \leq R-1$. Then, $U=\{v\}$ is a dominating set of $G^{R-1}$, and Lemma 2 implies the claim.

Thus, to improve the lower bound for MAXBD, one has to look to self-centered graphs. Moreover, if one wants to establish a lower bound of $\rho$, then a stable graph of minimum degree $\rho-1$ (from Corollary 1 ) is needed. Starting from these observations, we investigated the possibility to use small and suitably dense self-centered graphs as gadgets to build lower bound instances for increasing values of $R$. To illustrate the process, see Figure 2, where using a self-centered cubic graph of diameter 3 and size 20, we have been able to obtain a lower bound of 3 (it is not very hard to see that the obtained graph is in equilibrium).

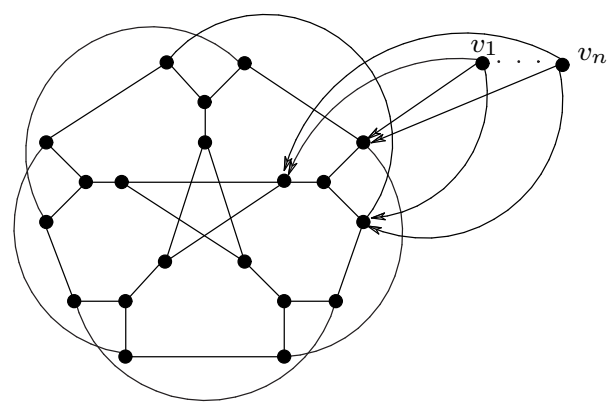

Fig. 2. A graph with $n+20$ nodes and $3 n+30$ edges, showing a lower bound for the PoA of MAXBD for $R=3$ approaching to 3 , as soon as $n$ grows. Edges within the gadget (on the left side) are bought by either of the incident nodes, while other edges are bought from the nodes they exit from.

Interestingly enough, the gadget is a famous extremal (i.e., maximal w.r.t. node addition) graph arising from the study of the degree-diameter problem, namely the problem of finding a largest size graph having a fixed maximum degree and diameter (for a comprehensive overview of the problem, we refer the reader to [1]). More precisely, the gadget is a graph of largest possible size having maximum degree $\Delta=3$ and diameter $R=3$. In fact, this seems not to be coincidental, since also Moore graphs (which are extremal graphs for $R=2$ and $\Delta=2,3,7,57$ ), and the extremal graph for $R=4$ and $\Delta=3$ (see [1]), can be shown to be in equilibrium, and then they can be used as gadgets (clearly, the lower bounds implied by Moore graphs for $R=2$ are subsumed by our result in Theorem 6 ). Notice that from this, it follows that we actually have a lower bound of 3 for the PoA of MAxBD also for $R=4$. So, apparently there could be some strong connection between the equilibria for MAxBD and the extremal graphs w.r.t. to the degree-diameter problem, and we plan in the near future to explore such intriguing issue.

\section{References}

1. http://www-mat.upc.es/grup_de_grafs/grafs/taula_delta_d.html/, Universitat Polit $\tilde{A} "$ cnica de Catalunya, Barcelona, Spain.

2. S. Albers, S. Eilts, E. Even-Dar, Y. Mansour, and L. Roditty, On Nash equilibria for a network creation game, in Proceedings of the 17th ACM-SIAM Symposium on Discrete Algorithms (SODA'06), ACM Press, 89-98, 2006. 
3. E. Anshelevich, A. Dasgupta, É. Tardos, and T. Wexler, Near-optimal network design with selfish agents, in Proceedings of the 35th Annual ACM Symposium on Theory of Computing (STOC'03), ACM Press, 511-520, 2003.

4. N. Alon, E.D. Demaine, M. Hajiaghayi, and T. Leighton, Basic network creation games, in Proceedings of the 22nd ACM Symposium on Parallelism in Algorithms and Architectures (SPAA'10), ACM Press, 106-113, 2010.

5. N. Alon and J.H. Spencer, The probabilistic method, John Wiley, New York, NY, 1992.

6. N. Baumann and S. Stiller, The price of anarchy of a network creation game with exponential payoff, in 1st International Symposium on Algorithmic Game Theory (SAGT'08), Lecture Notes in Computer Science 4997, Springer, 218-229, 2008.

7. E.D. Demaine, M. Hajiaghayi, H. Mahini, and M. Zadimoghaddam, The price of anarchy in network creation games, in Proceedings of the 36th annual ACM Symposium on Principles of Distributed Computing (PODC'07), ACM Press, 292-298, 2007.

8. S. Ehsani, M. Fazli, A. Mehrabian, S.S. Sadeghabad, M. Saghafian, S. Shokatfadaee, and M. Safari, On a bounded budget network creation game, in Proceedings of the 23rd ACM Symposium on Parallelism in Algorithms and Architectures (SPAA'11), ACM Press, in press, 2011.

9. A. Fabrikant, A. Luthra, E. Maneva, C.H. Papadimitriou, and S. Shenker, On a network creation game, in Proceedings of the 22nd Symposium on Principles of Distributed Computing (PODC'03), ACM Press, 347-351, 2003.

10. O. Kariv and S.L. Hakimi, An algorithmic approach to network location problems. II: The p-medians, SIAM J. Applied Math. 37(3):539-560, 1979.

11. E. Koutsoupias and C.H. Papadimitriou, Worst-case equilibria, in Proceedings of the 16th Annual Symposium on Theoretical Aspects of Computer Science (STACS'99), Lecture Notes in Computer Science 1563, Springer, 404-413, 1999.

12. N. Laoutaris, L.J. Poplawski, R. Rajaraman, R. Sundaram, and S.-H. Teng, Bounded budget connection (BBC) games or how to make friends and influence people, on a budget, in Proceedings of the 27th ACM Symposium on Principles of Distributed Computing (PODC'08), ACM Press, 165-174, 2008.

13. M. Mihalák and J.C. Schlegel, The Price of Anarchy in network creation games is (mostly) constant, in Proceedings of the 3rd International Symposium on Algorithmic Game Theory (SAGT'10), Lecture Notes in Computer Science 6386, Springer, 276-287, 2010. 